\title{
Numerical Integration Over the Triangle
}

\author{
By Gerald E. Bartholomew
}

1. Introduction. Integration formulae for numerical integration over the simplex in $n$-space have been given by Hammer, Marlowe, and Stroud [1]. This paper describes a method for the evaluation of integrals over a triangle, based on a formula developed in [1]. The mid-points of the sides of the triangle are used as the points of evaluation in this method.

Other possibilities may be used; for example, the trisection points of the medians that are not the centroid could be used as the points of evaluation. These points are symmetrically spaced and the weights, $w_{i}$, again are equal. The centroid could also be used as a single point of evaluation with $w_{i}$ equal to the area of the triangle. As another modification to this method, one could calculate the appropriate mid-points of the reference triangle for a reasonable number of subdivisions, then retain them as constants rather than calculate each point as it is used. Recently, (1958) Mr. Walter Leffin carried out computations by the use of these methods.

2. Mathematical Formulation. Consider the formula

$$
\int_{T} \int f(u, v) d u d v \doteq \sum_{i} w_{i} f\left(u_{i} v_{i}\right)
$$

where $T$ is a triangle, $w_{i}=A / 3$ for all $i, A$ being the area of $T$, and the points $\left(u_{i}, v_{i}\right)$ are the mid-points of the sides of $T$. Formula (1) then gives a first approximation to the integral. Now divide $T$ into four triangles by connecting the mid-points of the sides. Evaluate (1) over each subtriangle $T^{\prime}$ and sum. This gives a second approximation of the original integral. Iterate this process until the relation

$$
\left|I_{n}-I_{n-1}\right|<\mathcal{E}
$$

is satisfied for some prescribed $\varepsilon$, where $I_{n}$ is the $n$th approximation to the integral.

To find the desired mid-points of the sides of each triangle for successive subdivision, it was found convenient to subdivide a reference triangle $R$. Then the affine transformation

$$
\begin{aligned}
& u=a_{11} x+a_{12} y+a_{13} \\
& v=a_{21} x+a_{22} y+a_{23}
\end{aligned}
$$

will give the proper points of $T$ for the evaluation of (1). $R$ is defined by the vertices $(0,0),(1,-1),(1,1)$. The transformation (3) must then map $R \rightarrow T$ in the following manner

$$
\begin{aligned}
(0,0) & \rightarrow\left(u_{1}, v_{1}\right) \\
(1,-1) & \rightarrow\left(u_{2}, v_{2}\right) \\
(1,1) & \rightarrow\left(u_{3}, v_{3}\right)
\end{aligned}
$$

Received August 16, 1957; revised June 9, 1959. The machine calculation was supported by Army Ordnance. 
where $\left(u_{1}, v_{1}\right),\left(u_{2}, v_{2}\right),\left(u_{3}, v_{3}\right)$ are the vertices of $T$. Substituting (4) into (3) and solving for the $A_{i j}$, we obtain

$$
\begin{gathered}
a_{11}=\frac{u_{2}+u_{3}-2 u_{1}}{2} \quad a_{21}=\frac{v_{2}+v_{3}-2 v_{1}}{2} \quad a_{12}=\frac{u_{3}-u_{2}}{2} \\
a_{22}=\frac{v_{3}-v_{2}}{2} \quad a_{13}=u_{1} \quad a_{23}=v_{1} .
\end{gathered}
$$

Since the area of $R$ is one unit, the absolute value of the Jacobian of (3) is the area of $T$. Note, also, that the area of each $T^{\prime}$ is one-fourth of the area of $T$. Hence the $w_{i}$ are easily determined for each approximation.

The mid-points of the triangles in the previous $(n-1)$ subdivisions together with their vertices constitute the vertices of the triangles of the $n$th subdivision. Hence one could find the mid-points for the $n$th subdivision by use of the formulae of analytic geometry. This is undesirable because of the large amount of information that must be retained. For example, the 5th approximation has $768 \mathrm{mid}$ points, 408 of them are distinct. Therefore, a procedure is used that greatly reduces the amount of information that must be retained after each subdivision. This method uses three incremental values, $\Delta_{1}, \Delta_{2}, \Delta_{3}$, and the previously calculated mid-point, along with certain features of the triangle $R$ to determine the next mid-point.

Since $R$ is symmetrical about the $x$-axis, by reflection across the $x$-axis one generates a point $p_{i}{ }^{*}=\left(x_{i},-y_{i}\right)$ for every point $p_{i}=\left(x_{i}, y_{i}\right)$, where $y \neq 0$. Also every mid-point not lying on the boundary of $R$ is a mid-point of two triangles. Hence one must check whether $(a) x=|y|$ or $(b) x=1$ to determine whether it is an interior point with respect to $R$. The increments $\Delta_{1}, \Delta_{2}, \Delta_{3}$ have
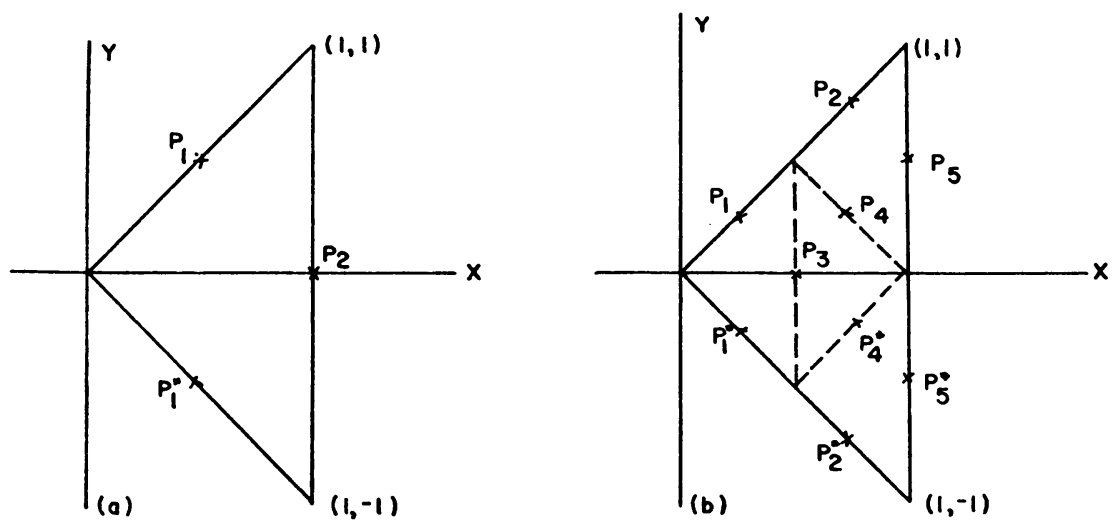

FIG. 1.-The sequence of point generation for first and second subdivisions. 
the initial values $\frac{1}{2}, 1,0$ respectively. $\Delta_{1}$ and $\Delta_{2}$ are divided by a factor, two, for each successive subdivision, while $\Delta_{3}$ is altered during each subdivision and is reset to zero at the start of the next subdivision.

The flow diagram in section 3 will show specifically how the increments are used to generate the $p_{i}$. Figure 1 shows the order in which the points are generated for the first and second subdivisions. The points $p_{i}$ are actually calculated along straight lines originating at the $x$-axis with slope one. Hence the obvious check must be made to see whether the next point should be calculated along the same line, or whether the next point should initiate the next line.

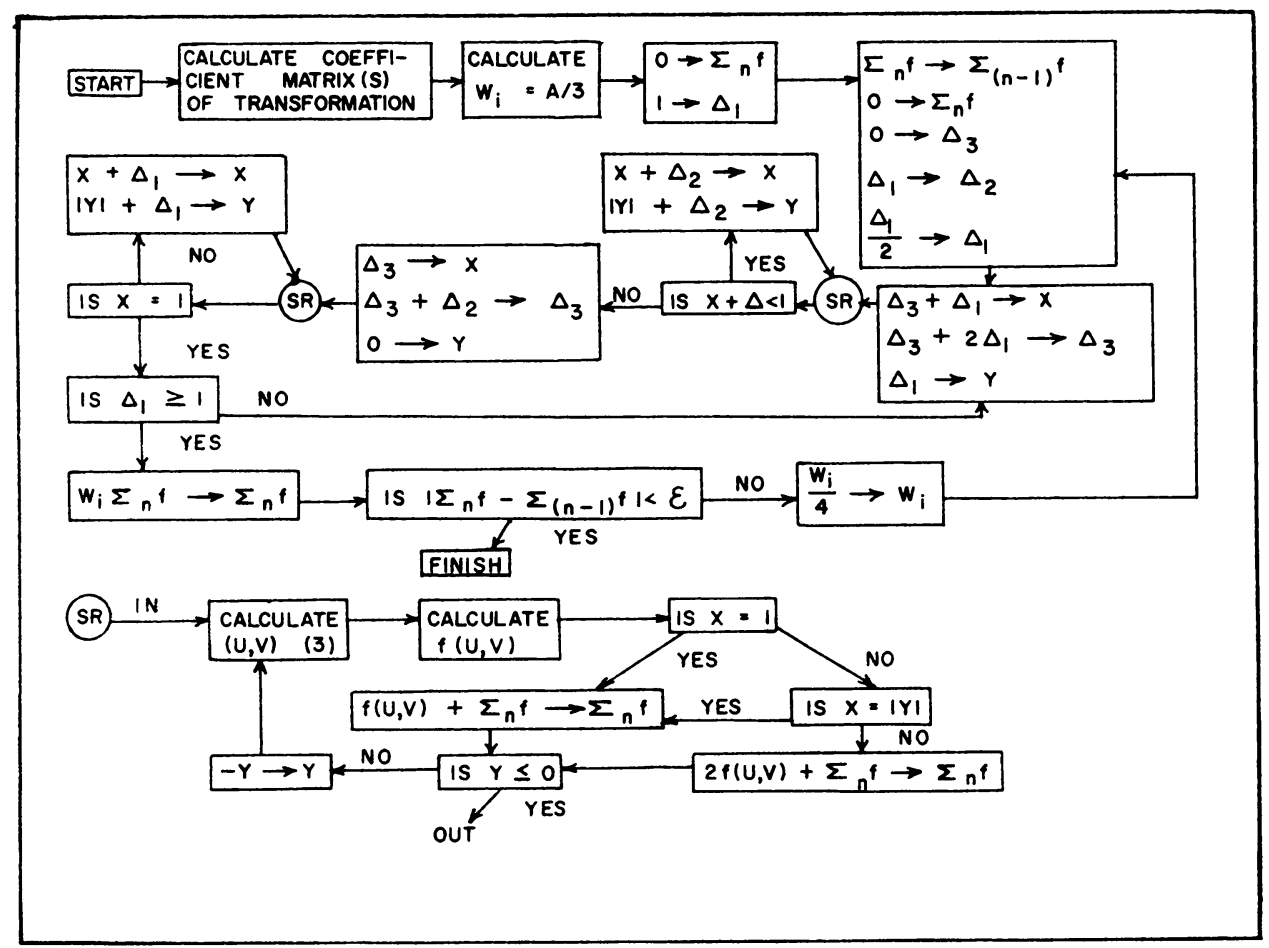

Fig. 2.-Flow diagram.

TABLE 1

\begin{tabular}{|c|c|c|c|c|c|}
\hline$f$ & Triangle & $I$ & $n$ & $I_{n}$ & $\varepsilon$ \\
\hline$x^{3}+y^{3}$ & $(0,0), \underset{R}{(2,0),}(2,1)$ & $\begin{array}{l}3.3000 \\
.52323\end{array}$ & $\begin{array}{l}6 \\
2\end{array}$ & $\left|\begin{array}{c}3.3000009 \\
.52316017\end{array}\right|$ & $\begin{array}{l}10^{-5} \\
10^{-3}\end{array}$ \\
\hline $\begin{array}{l}2+y \\
\sin x \\
1\end{array}$ & $R$ & .60234 & 3 & .60232905 & $10^{-3}$ \\
\hline $\begin{array}{l}\overline{\sqrt{x}} \\
f=\left\{\begin{array}{l}1 \text { for } x-y<\frac{1}{2} \\
2 \text { for } x-y \geqq \frac{1}{2}\end{array}\right.\end{array}$ & $\begin{array}{l}R \\
R\end{array}$ & $\begin{array}{l}1.3333 \\
1.5625\end{array}$ & $\begin{array}{l}4 \\
4\end{array}$ & $\begin{array}{l}1.3307964 \\
1.5937500\end{array}$ & $\begin{array}{l}10^{-2} \\
10^{-1}\end{array}$ \\
\hline
\end{tabular}


3. Flow diagram. Figure 2 contains the flow diagram. The notation used in the flow diagram should be explained. The expression $a \rightarrow b$ means that $a$ replaces $b$. For example, $1 \rightarrow \Delta_{1}$ means that $\Delta_{1}$ is set equal to one. $\sum_{n} f$ is the partial calculation of $I_{n}$ that has already been made, or it is $I_{n}$ if the calculation is completed for the $n$th approximation. $\sum_{(n-1)} f$ is then $I_{n-1}$.

4. Results of Trial Calculations. A code was devised for the IBM 650 and several trial calculations were run. Table 1 gives the results that were obtained. It might be pointed out that formula (1) is exact in the first approximation for a quadratic function. This problem was suggested by Professor Preston C. Hammer.

University of Wisconsin,

Madison 6, Wisconsin

1. P. C. Hammer, O. J. Marlowe \& A. H. Stroud, "Numerical integration over simplexes and cones," MTAC, v. 10, 1956, p. 130-137. 